\title{
Magnetic Orientation of Side-Chain Liquid Crystalline Polyguanamines
}

\author{
Yoshiyuki Oishi, Takahide Suzuki, Hiroshi Awano, and Koichiro Yonetake
}

\begin{abstract}
Novel side-chain liquid crystalline polyguanamine with mesogenic groups (LCPGs) exhibited smectic liquid crystallinity. The polymers had an oriented structure under a magnetic field due to higher diamagnetic susceptibility of the mesogenic group. The mesogenic groups could be per pendicularly arranged on a glass substrate under a vertical magnetic field. Furthermore, they were obliquely aligned on the glass substrate by a tilt magnetic field. Tilt angles between the mesogen and the glass substrate were almost consistent with the directions of applied magnetic fields. Magnetic fields afforded various oriented structures, and the magneto-oriented structures were fixed in the samples.
\end{abstract}

Index Terms-Magnetic field, magnetic orientation, magneto-oriented structure, mesogenic group, side-chain crystalline polymer.

\section{INTRODUCTION}

$\mathbf{P}$ OLYGUANAMINES can be readily modified with various functional groups, because it is easy to introduce them on triazine rings in the main chain [1]. The polyguanamine modified with mesogenic groups would exhibit a liquid crystalline nature, and its fine structure is easily controlled using external fields such as electric fields, mechanical fields, polarized UV light fields, or magnetic fields. Particularly, the magnetic fields can arrange the mesogenic groups in all directions.

In this study, novel side-chain liquid crystalline polyguanamines were used for magnetic orientation. A magnetic field was applied parallel, vertically, or obliquely to the liquid crystalline polyguanamine. Magneto-oriented structures in the polymer samples were analyzed by using polarizing optical microscopy (POM) and X-ray diffraction (XRD) methods.

\section{EXPERIMENTAL}

\section{A. Polymer Sample}

Liquid crystalline polyguanamines (LCPGs) with a cyanobiphenyl or methoxybiphenyl mesogenic group were synthesized according to the literature [2] and shown in Figs. 1 and 2.

\section{B. Measurement}

Differential scanning calorimetry (DSC) was performed by a DSC 200 (Seiko I\&E Ltd.) under nitrogen at a heating and

Manuscript received October 21, 2003.

Y. Oishi is with the Department of Chemical Engineering, Iwate University, Morioka, 020-8551, Japan (e-mail: yoshiyu@iwate-u.ac.jp).

T. Suzuki, H. Awano, and K. Yonetake are with the Department of Polymer Science and Engineering, Yamagata University, Yonezawa 992-8510, Japan (e-mail: yone@yz.yamagata-u.ac.jp).

Digital Object Identifier 10.1109/TASC.2004.831009

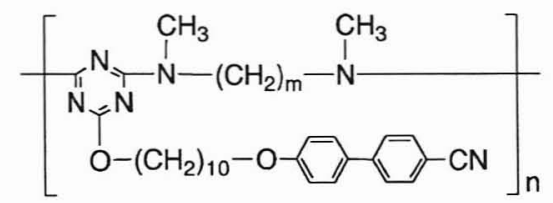

Fig. 1. Chemical structure of LCPG-1 $(m=6)$, and LCPG-2 $(m=12)$.

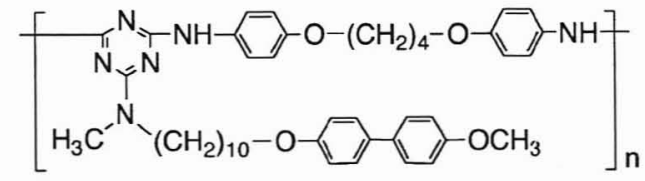

Fig. 2. Chemical structure of LCPG-3.

cooling rate of $10^{\circ} \mathrm{C} / \mathrm{min}$. Optical textures of the samples during a heating and cooling process were obtained using a polarizing optical microscope (POM: Olympus BX-50P) equipped with a hot stage (Linkam TH-600RMS) under nitrogen. X-ray diffraction experiments were carried out by a RAD-rA diffractometer (Rigaku Denki Co. Ltd). Nickel-filtered $\mathrm{CuK} \alpha$ radiation was employed. X-ray diffraction (XRD) profiles were measured by a scintillation counter system with a $1.0 \mathrm{~mm}$-diameter pinhole collimator and $1 \times 1^{\circ}$ receiving slit using a step scanning method $\left(0.05^{\circ}\right.$ steps every 4 seconds). The XRD photographs were taken by a flat Laue camera with a $0.5 \mathrm{~mm}$-diameter pinhole collimator.

\section{Magnetic Orientation}

The samples were heated up to their isotropic melt states, and the magnetic field was applied to them during cooling at a rate of $1{ }^{\circ} \mathrm{C} / \mathrm{min}$. The fields of $2.4 \mathrm{~T}$ and $10 \mathrm{~T}$ were generated by an electromagnet TM-WV8615MRC-156 (Tamagawa Co.) and a superconducting magnet JMTD-10 T 100 M (Japan Magnet Technology), respectively.

\section{RESULTS AND DISCUSSION}

\section{A. Structure of Polyguanamines}

The phase transition temperatures of polyguanamines were determined by DSC. LCPG-1 exhibited single endothermic peak at $92.9^{\circ} \mathrm{C}$ in the heating DSC thermogram shown in Fig. 3. Above $92.9^{\circ} \mathrm{C}$, it became isotropic melt state, because dark field was observed under POM with crossed polarizers. A typical schlieren texture due to nematic phase appeared around $90.7^{\circ} \mathrm{C}$ on cooling. The schlieren texture became tiny on further cooling. Thus, a higher ordered liquid-crystalline structure was formed at room temperature. DSC thermograms of LCPG-2 exhibited single peak on heating and two peaks on cooling as shown in Fig. 4. A schlieren texture appeared 

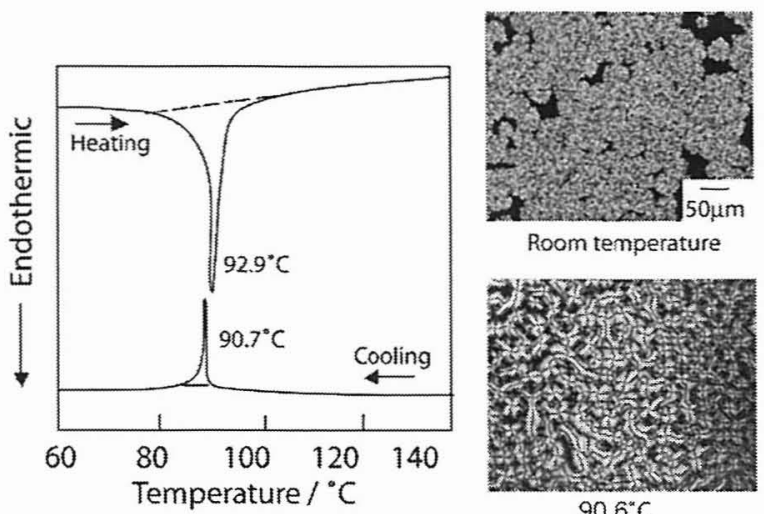

$90.6^{*} \mathrm{C}$

Fig. 3. DSC thermograms and optical textures of LCPG-1.
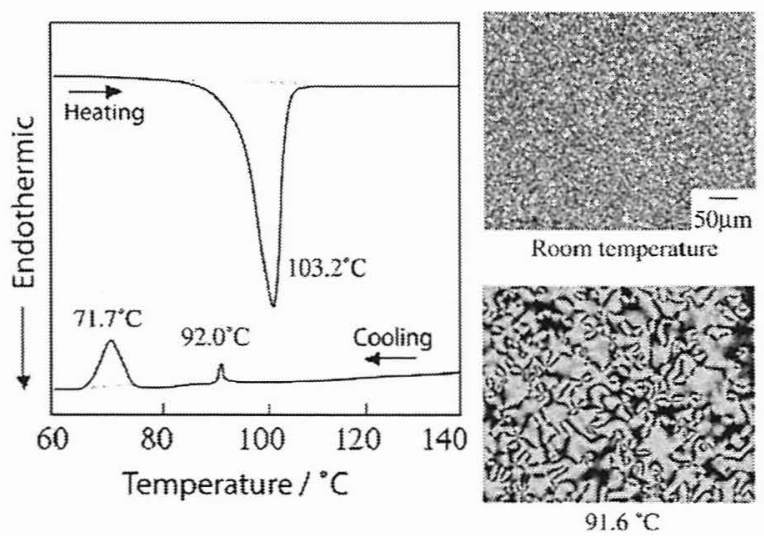

Fig. 4. DSC thermograms and optical textures of LCPG-2.

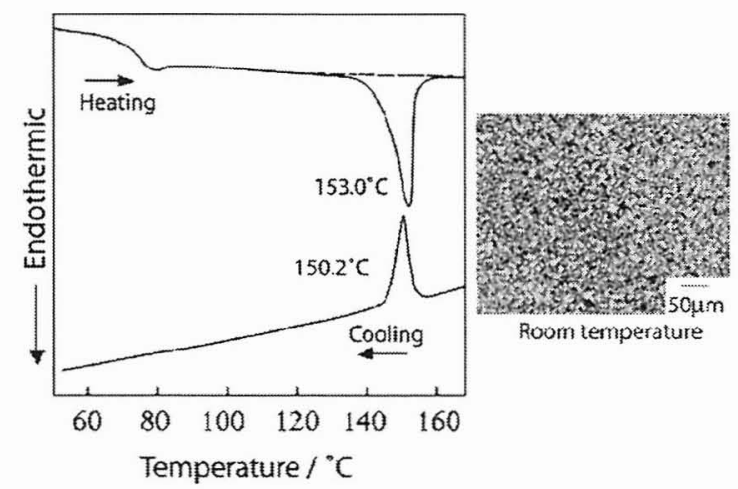

Fig. 5. DSC thermograms and optical texture of LCPG-3.

around $92^{\circ} \mathrm{C}$ and a tiny texture was observed below $71.7^{\circ} \mathrm{C}$; two liquid crystalline phases formed on cooling. Thus, LCPG-2 is an enantiotropic liquid crystal showing nematic and smectic liquid crystalline phases on cooling. DSC thermograms and optical texture of LCPG-3 are shown in Fig. 5. Single peak was observed around $150^{\circ} \mathrm{C}$ on the heating and cooling scans. The phase transition of LCPG-3 is similar to that of LCPG-1.

Fig. 6 shows XRD traces of LCPG-1, LCPG-2, and LCPG-3 measured at room temperature. The XRD pattern of LCPG- 1 has three periodic peaks at low-angle area, which are characteristic of smectic layer structure, and a diffuse reflection at wide-angle area. The $d$-spacings of the periodic peaks at low-angle area were $2.90 \mathrm{~nm}, 1.52 \mathrm{~nm}$, and $1.11 \mathrm{~nm}$, which were assigned to 1st, 2nd, and 3rd reflections, respectively. Thus, the liquid crystalline phase of LCPG-1 is smectic B at room temperature. From the XRD trace of LCPG-2, the periodic peaks at low-angle area

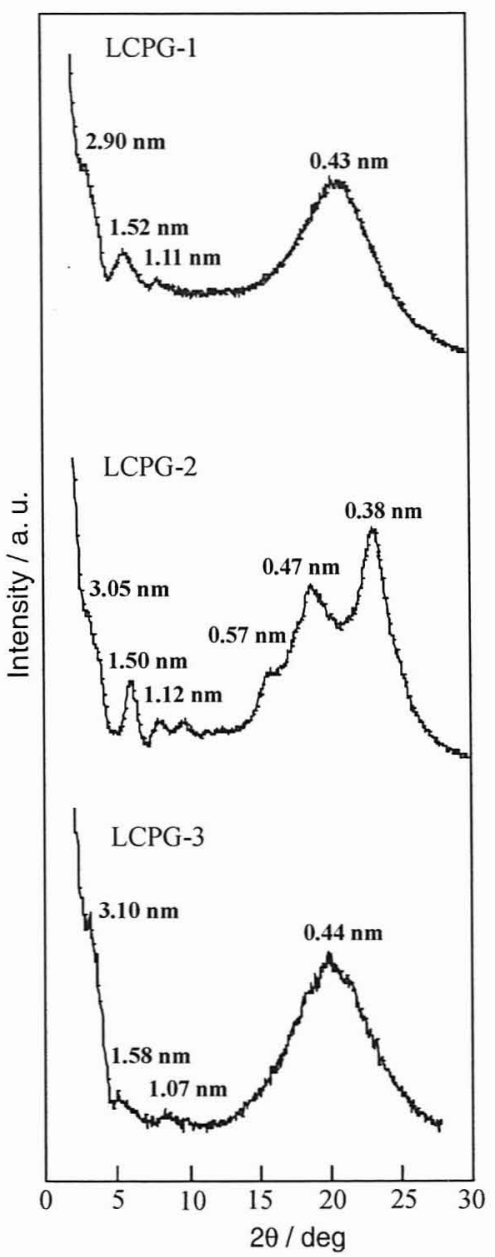

Fig. 6. XRD traces of LCPG-1, LCPG-2, and LCPG-3.

and comparatively sharp reflections at wide-angle area were observed. The smectic phase of LCPG-2 at room temperature is likely assigned to smectic E. The XRD profile of LCPG-3 is similar to that of LCPG-1. The liquid crystalline phase of LCP-3 is also smectic B. The layer spacings of LCPG-1, LCPG-2, and LCPG-3 were determined to be $2.90,3.05$, and $3.10 \mathrm{~nm}$, respectively. They were consistent with the molecular lengths of the side mesogenic groups, so that their smectic layer structure is monolayer.

\section{B. Magnetic Orientation of Polyguanamines}

A magnetic field of $10 \mathrm{~T}$ was applied parallel to the sidechain liquid crystalline polyguanamines, LCPG-1, LCPG-2, and LCPG-3 during cooling at $1{ }^{\circ} \mathrm{C} / \mathrm{min}$. The optical textures observed under crossed polarizers are shown in Fig. 7. Extended textures are observed in LCPG-1 and LCPG-2. A streak texture is clearly observed in LCPG-1, where the schlieren texture was extended in the magnetic field direction. There is no conspicuous change in the optical texture of LCPG-3. However, the XRD photograph of LCPG-3 exhibited an oriented pattern as shown in Fig. 8. The orientation coefficient was estimated to be 0.74 . The mesogenic groups were highly oriented to the magnetic field, and the smectic layer was formed vertically to the magnetic field. Thus, liquid crystalline polyguanamines (LCPGs) could be oriented by magnetic field as well as other side-chain liquid crystalline polymers [3]. 

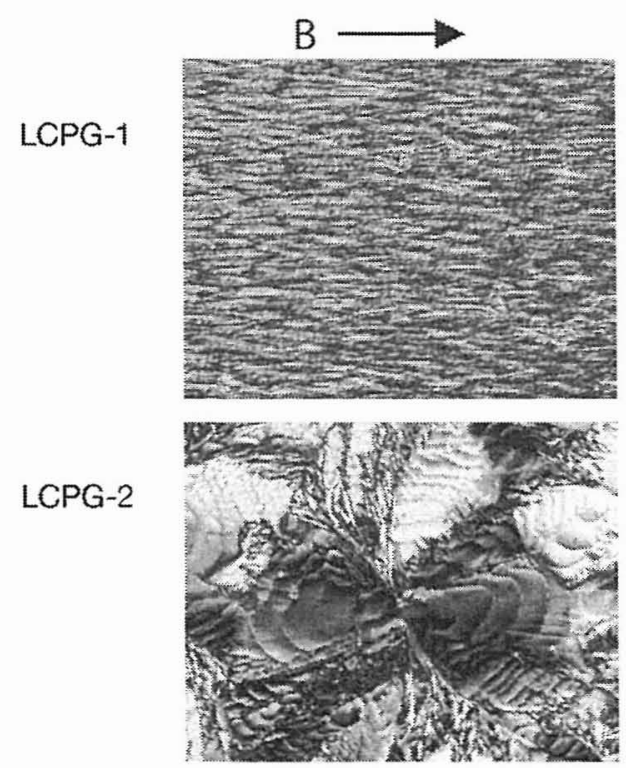

LCPG-3

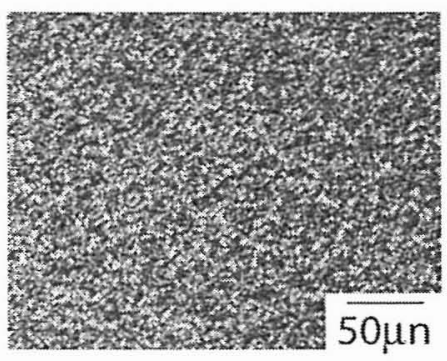

Fig. 7. Optical textures of magneto-oriented LCPGs. The magnetic field of 10 $\mathrm{T}$ was applied parallel to the samples.

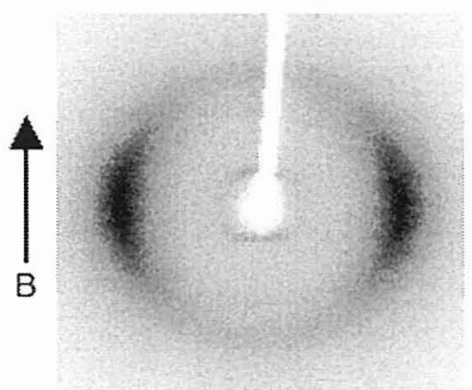

Fig. 8. XRD pattern of magneto-oriented LCPG-3. Magnetic field was $10 \mathrm{~T}$.

The magnetic field was applied vertically to the polymer sample on the glass substrate. The magneto-oriented samples exhibited a dark field under POM with crossed polarizers, and a conoscopic image obtained at the dark area was a typical isogyre pattern as shown in Fig. 9. The mesogenic groups were perpendicularly ordered on the glass substrate, because a cross point of the isogyre pattern lied in the center of the conoscopic image. A homeotropic structure was formed in the sample. Thus, the mesogenic groups could be arranged parallel to the magnetic field direction.

The magnetic fields were applied to the samples at tilt angles of 30,45 , and 60 degree in $2.4 \mathrm{~T}$ and $10 \mathrm{~T}$, respectively. Optical textures and conoscopic images of the magneto-oriented samples are shown in Fig. 10. Dark and small optical textures appeared in the sample to which the magnetic field was obliquely applied at 30 degree. In the conoscopic image observed in the area, the cross point of the isogyre pattern greatly deviated to the
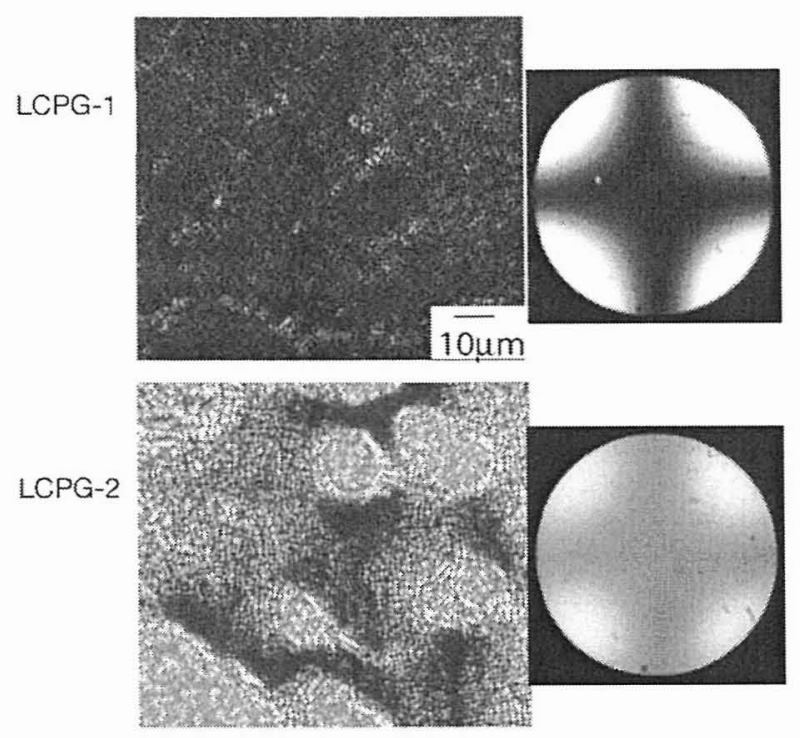

LCPG-3

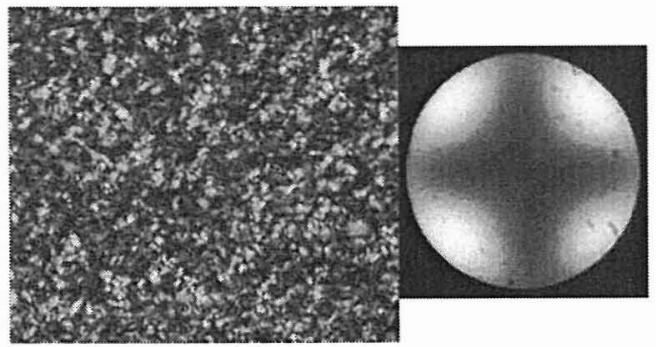

Fig. 9. Optical textures and conoscopic images of magneto-oriented samples observed under POM with crossed polarizers. The magnetic field of $10 \mathrm{~T}$ was applied perpendicular to the samples.

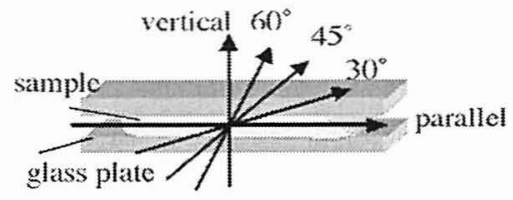

$2.4 \mathrm{~T}$

$10 \mathrm{~T}$
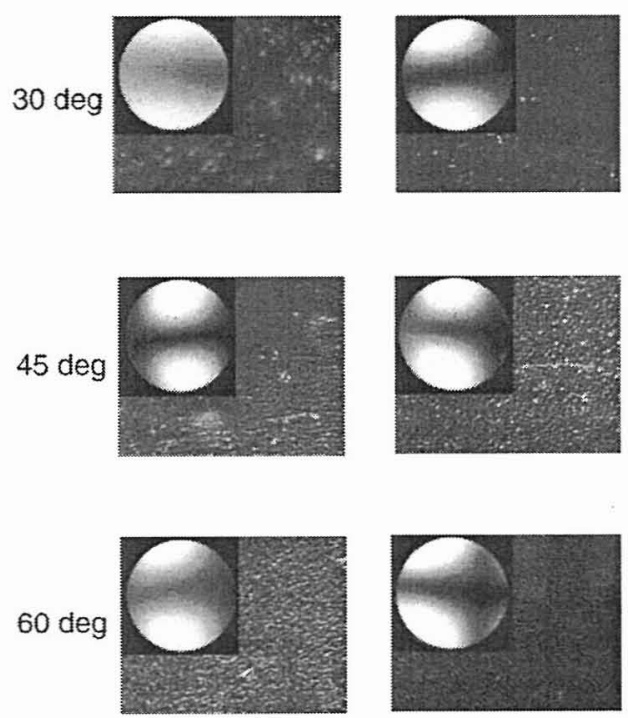

Fig. 10. Optical textures and conoscopic images of magneto-oriented LCPG-1 observed under POM with crossed polarizers. The magnetic fields were 2.4 and $10 \mathrm{~T}$. The setting angles were 30,45 , and 60 degree.

right side. This suggests that the mesogenic groups were tilted to the right side on the glass substrate. Similar optical textures 


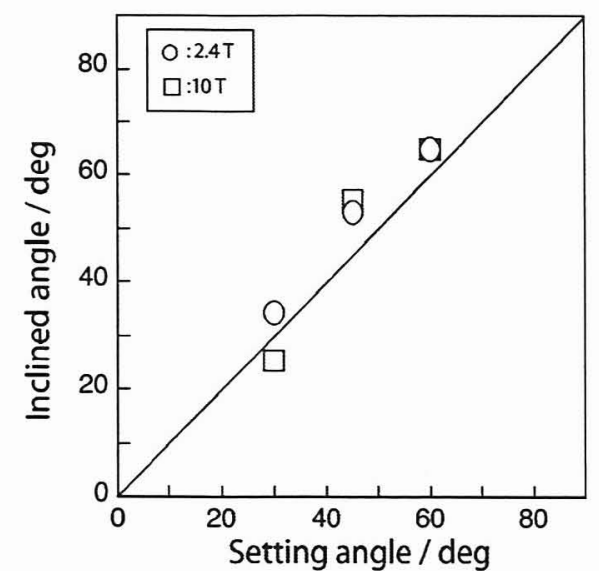

Fig. 11. Relationship between inclined angle of mesogen of LCPG-1 and setting angle of magnetic field direction. The magnetic fields were 2.4 and $10 \mathrm{~T}$.

and conoscopic images of magneto-oriented samples were obtained at the tilt angles of 45 and 60 degree. The deviation of the cross point of the isogyre pattern increased with decreasing the setting angle of the magnetic field direction.

Furthermore, interference colors of the samples observed under POM with sensitive color plate $(\lambda=530 \mathrm{~nm})$ have changed; their retardation became larger with decreasing the setting angle. These results confirmed that the mesogenic groups of LCPGs were aligned to the magnetic field direction.

An inclined angle between the mesogenic group and the glass substrate were measured by using POM. Fig. 11 represents the relationship between the inclined angle and the setting angle. The inclined angles were almost consistent with the setting angles. The mesogenic groups of LCPG-1 were aligned parallel to the magnetic field applied, and the tilt structures were successfully fixed on the glass substrates. Similar results were obtained in other polymeric liquid crystalline materials.

\section{CONCLUSION}

Novel side-chain liquid crystalline polyguanamines (LCPGs) with mesogenic side groups exhibited smectic liquid crystalline phase. The cooling LCPGs in a magnetic field from the isotropic to smectic phase resulted in a magnetic orientation of the mesogenic groups of LCPGs. The homogeneous, homeotropic, and tilt oriented structures were successfully obtained on the glass substrates. Therefore, it could be possible to enhance the optical and electrical properties of polymeric materials by using the magnetic orientation technique.

\section{REFERENCES}

[1] Y. Oishi, "Synthesis of functional condensation polymers containing triazine rings," Kobunshi, vol. 48, pp. 274-278, 1999.

[2] M. Nomura, Y. Oishi, H. Arai, H. Hirahara, K. Mori, and K. Yonetake, "Synthesis of triazine containing polymers with mesogenic groups," Polym. Prepr., Jp., vol. 50, p. 219, 2001.

[3] D. Ferri et al., "Phase and orientation behaviors in liquid crystalline main-chain/side-group block copolymers," J. Polym. Sci., Part B, Polym. Phys., vol. 36, pp. 21-29, 1998. 